\title{
CRITICAL ANALYSIS OF SOME CRITICISMS OF THE DROPLET MODEL
}

\author{
H. Krivine \\ Division de Physique Théorique*, Institut de Physique Nucléaire, \\ $B P 1,91406$ Orsay Cedex, France
}

\begin{abstract}
Résumé - Nous montrons que dans un systēme nucléaire semi infini la formule du modèle de la gouttelette liquide reliant la tension de symétrie de surface à la peau de neutron est nécessairement vérifiée, pourvu qu'il existe une densité d'hamiltonien dépendant seulement des densités (et de leurs dérivées). on effectue ensuite un calcul auto-cohërent dans une plaque. Nous faisons finalement quelques remarques pour le cas des noyaux finis.

Abstract - We prove that in the semi infinite nuclear system the droplet model formula relating the surface symmetry tension to the neutron skin is satisfied provided there exists an hamiltonian density involving only the densities and its derivatives. We make a fully self consistent calculation in a slab. We finally make some comments about the case of finite nuclei.
\end{abstract}

\section{I - INTRODUCTION}

As well known, the knowledge of the surface symmetry energy $E_{S S}$ is relevant for the calculation of fission barriers, heavy ion collisions, but also for understanding the strength distribution shape of $T=1$ resonances. It is also relevant for some as trophysics equilibrium calculations.

Unfortunately, ESS remains rather undetermined both from experimental data as from theoretical calculations based on microscopic interactions. One of the major interests of the Droplet Mode 1 Theory (D.M.)/1/ from this point of view is to relate simply the surface symmetry tension $\sigma_{\delta}\left(4 \pi r_{0}{ }^{2} \sigma_{\delta}=E_{S S}\right.$, where $r_{0}$ is the nuclear matter radius) to the neutron skin $t$.( $t$ is a geometrical quantity related -but not directly - to the difference between the neutron and proton rms). This relation can be written in a nucleus

$$
\sigma_{\delta}=\frac{1}{2} \mathrm{Jp} n \mathrm{~nm} \frac{\mathrm{t}}{\delta}
$$

3 is the symmetry energy of the nuclear matter with the density $\rho_{n m}$, in the absence of coulomb force, $\bar{\delta}$ is the value at the center of the local asymetry $\delta$

$$
\delta=\frac{\rho_{n}-\rho_{p}}{\rho_{n}+\rho_{p}}
$$

In the semi infinite matter, $\bar{\delta}=I$ (the usual $n-p$ asymmetry coefficient). The proof of this relation given by Myers and Swiatecki is rather subtle. This is

\footnotetext{
* Laboratoire Associē au C.N.R.S.
} 
perhaps why it was never criticized on theoretical grounds ; however some numerical results have been found, which contradict the D.M. theory in this respect $12 /$. In this work, we will first concentrate on another derivation of the formula (1) in a simple case (semi-infinite nuclear matter or slab, no Coulomb energy). We will prove that the original result is rigorously exact under the two - rather general-assumptions

i) the nuclear forces are saturating

ii) the energy of a fermion system in mutual interaction can be described by an Hamiltonian density $\mathcal{H}$ depending only upon the diagonal one-body density matrix. In the first part of this work, we derive and analyze eq.(1). In the subsequent section we give an example of calculation of $\sigma_{\delta}$. Finally we discuss some problems occuring when one takes the case of the finite nuclei.

\section{II - DERIVATION OF THE D.M. FORMULA}

The main difficulty to follow the original demonstration of Myers and Swiatecki lies in the fact that their starting point is a finite system with $A$ nucleons (Coulomb) force included), while the final formulae deal only with properties of semi-infinite system. As a result, they often deal with quantities that remain finite, as products of factors going to zero like $\left(1-\frac{\bar{\delta}}{I}\right)$ and to infinity with the surface of the nucleus. Therefore we think that it is simpler to consider the case of the slab of nuclear matter, avoiding any limiting case.

\section{II - 1. Background and notation}

One writes

$$
\alpha=\rho_{n}-\rho_{p}=\rho \delta
$$

and one supposes the matter "on the left"

$$
\begin{aligned}
& \rho_{n}(-\infty)=\frac{1}{2} \rho_{n m}(1+I) \\
& \rho_{p}(-\infty)=\frac{1}{2} \rho_{n m}(1-I)
\end{aligned}
$$

Though the sharp equivalent radii $R_{n}$ and $R_{p}$ are meaningless, their difference, which is the neutron skin, $t$, is finite and defined by

$$
t=R_{n}-R_{p}=\int\left(\frac{\rho_{n}}{\rho_{n}(-\infty)}-\frac{\rho_{p}}{\rho_{p}(-\infty)}\right) d x
$$

Using then eq.(3) one gets to order $I^{2}$

$$
t=\frac{2 I}{\rho_{n m}} \int\left(\frac{\alpha}{I}-\rho\right) d x .
$$

Let us now split the Hamiltonian density into its isoscalar and isovector part

$$
\mathcal{H}=\mathscr{H}_{\rho}+\mathcal{H}_{\alpha}
$$

The isospin symmetry allows to expand $\mathfrak{H}_{\alpha}$

$$
\mathscr{H}_{\alpha}=\mathcal{A}\left(\rho, \bar{\nabla}_{\rho} . .\right) \alpha^{2}+B\left(\rho, \bar{\nabla}_{\rho} . .\right)\left|\nabla_{\alpha}\right|^{2}+. .
$$

(For the sake of simplicity we restrict ourselves to the first two terms of the 
expansion although the next terms which contain higher order derivatives would not alter the following demonstration).

II - 2. Proof

In the absence of any constraint, the proton and neutron densities are identical. To get an asymmetry one had to impose

$$
\int\left(\frac{\alpha}{I}-\rho\right) d x=c^{\text {te }}
$$

The constraint (7) implies that the local asymptotic asymmetry "on the left" is equal to a fixed number I. Eq. (7) is, in a one dimensional space, the counter part of the 3 dimensional constraint

$$
\int\left(\frac{\alpha}{I}-\rho\right) d \bar{r}=0
$$

(which simply means that $\frac{\mathrm{N}-\mathrm{Z}}{\mathrm{T}}-\mathrm{A}=0$ ). Eqs.(5) and (7) show that imposing an asymmetry $I$ in the semi infinite huclear matter is equivalent to fixing the value of the neutron skin (over I). Denoting by $\left(\frac{E}{A}\right)_{0}$ the energy per particle in the asymmetrical nuclear matter and $\left(\frac{E}{A}\right)_{n m}$ the corresponding quantity when $I=0$, we have

$$
\left(\frac{E}{A}\right)_{0}=\left(\frac{E}{A}\right)_{n m}+J I^{2} \text {. }
$$

At equilibrium, the quantity

$$
\Sigma(I)=\int\left[\mathbb{H}-\left(\frac{E}{A}\right)_{0} \rho-\mu\left(\frac{\alpha}{I}-\rho\right)\right] d x
$$

must be stationary against any variation of $\rho$ and $\alpha$. With respect to $\alpha$ the Euler Lagrange equation gives (see eq.(6))

$$
2 A \alpha \alpha-2 B^{\prime} \alpha^{\prime}-2 B \alpha^{\prime \prime}=\frac{H}{I} \text {. }
$$

Since $\mu$ is a constant, it can be evaluated for $x=-\infty$, where $\alpha^{\prime}=\alpha "=0, \alpha=I \rho_{n m}$ and

$$
\mathcal{H}_{\alpha}(-\infty)=I^{2} \rho_{n m}^{2} A(-\infty)=I^{2} J \rho_{n m}
$$

so that we have

$$
\mu=2 J I^{2} \text {. }
$$

On the other hand, the surface tension $\sigma$ of the slab is defined by :

$$
\sigma=\int\left[\mathcal{H}-\left(\frac{E}{A}\right)_{0} \rho\right] d x
$$

It should be noted that the difference between the surface tension $\sigma$ (eq.(13)) and $\sum(\mathrm{I})$ (eq. (10)) is

$$
\begin{aligned}
\sigma-\sum(I) & =2 J I^{2} \int\left(\frac{a}{I}-\rho\right) d x \\
& =J I t \rho_{n m}
\end{aligned}
$$

This corresponds to the tension due to the constraint. We are now on a position to 
prove relation (1). In the semi infinite system, Eq.(1) reads

$$
\sigma_{\delta}=\frac{1}{2} \frac{d^{2}}{d I^{2}} \sigma=\frac{1}{2} j \rho_{n m} \frac{t}{I} \text {. }
$$

Since up to terms of order $I^{2}, \frac{\alpha}{I}$ is I-independent, the hamiltonian

$$
\mathscr{H}=\mathscr{H}_{\rho}+I^{2}\left[\hat{\omega} \frac{\alpha^{2}}{I^{2}}+B \frac{\left|\nabla_{\alpha}\right|^{2}}{I^{2}}\right]
$$

can be differentiated with respect to I and Eq.(15) is transformed into

$$
\sigma_{\delta}=\frac{1}{2} \frac{d^{2}}{d I^{2}} \int\left[\mathcal{H}-\left(\frac{E}{A}\right)_{0} \rho\right] d x=\int\left(\omega \frac{\alpha^{2}}{I^{2}}+B \frac{\left|\nabla_{\alpha}\right|^{2}}{I^{2}}-J \rho\right) d x
$$

Returning now to the Euler Lagrange equation (11) where we replace $\mu$ by its value, $2 \mathrm{JI}$, at equilibrium, we multiply both sides by $\alpha$, subtract $2 \mathrm{I}^{2} \mathrm{~J} \rho$ and integrate by parts to obtain

$$
2 \int\left(\Delta \alpha^{2}+B\left|\vec{\nabla}_{\alpha}\right|^{2}-I^{2} J \rho\right) d x=2 J I^{2} \int\left(\frac{\alpha}{I}-\rho\right) d x
$$

This relation together with Eq.(16) proves the D.M. relation (15).

We now make some comments on the above derivation. The second derivative of both sides of the Eq.(14a) gives (using Eq.(15))

$$
\frac{1}{2} \frac{d^{2} \Sigma}{d I^{2}}=\sigma_{\delta}-2 \sigma_{\delta}=-\sigma_{\delta}
$$

Eq.(17) shows that the symmetry energy per unit area taking into account the energy due to the constraint is exactly opposed to the symmetry surface tension $\sigma_{\delta}$ (which is $>0)$. This is so because the symmetry tension due to the constraint is exactly equal to $-2 \sigma_{\delta}$. The factor 2 is not accidental, but related to the quadratic dependence of $\mathcal{H}$ on $\alpha$.

When dealing with a mass formula for finite nuclei, the surface symmetry coefficient $\varepsilon_{\text {ss }}$ ( $i . e .$, the coefficient of $I^{2} A^{-\frac{1}{3}}$ in the expansion of $\frac{E}{A}$ ) must be calculated from $\frac{1}{2} \frac{\mathrm{d} \Sigma}{\mathrm{dI}^{2}}$. It is negative as a result of the balance between the surface symmetry energy $(>0)$ and the loss of energy due to the reshaping of the densities responsible of the formation of a neutron skin (or equivalently, in finite nuclei, because of the conservation of $\mathrm{N}-\mathrm{Z}$, a decreasing of $\delta(r)$ in the bulk of the nucleus from I to $\bar{\delta})$. Since $\alpha=\rho \delta$, Eq.(15) may be written

$$
\sigma_{\delta}=J \int\left(\frac{\delta}{I}-1\right) \rho d x
$$

and appears as the symmetry energy of nuclear matter per unit area weighted by the average of the excess of local asymmetry $\left(\frac{\delta}{1}-1\right) \rho$. Clearly any approximation which ignores the neutron skin (like the standard one : $\rho_{n}=\frac{N}{A} p, \rho_{p}=\frac{Z}{A} \rho$ ) is meaningless for such calculations. In a previous work $/ 3 /$ we have derived the approximate relation

$$
\sigma_{\delta}=2 \int\left[J-\varepsilon_{\delta}(\rho)\right] \rho d x
$$


where $\varepsilon_{\delta}(\rho)$ represents the volume part of $\frac{\mathscr{H}_{\alpha}}{\mathrm{I}^{2} \rho}$ (i.e., the part independent of the derivatives with respect to $\alpha$ ). In Eq. (19) $\sigma_{\delta}$ also appears as an average over the surface region of the defect of symmetry energy relative to the volume symmetry energy. It should be stressed that formula (19) is only a rough approximation ; its advantage as compared to the exact relation (18) is that it does not require the knowledge of the equilibrium densities in the asymmetrical slab. We now show how $\sigma_{\delta}$ in Eq.(18) can be evaluated.

\section{I - CALCULATION OF THE SURFACE SYMMETRY TENSION}

II I - 1. Method

We construct our density hami Ttonian $\mathcal{T}$ using Skyrme forces and some crude variant of the Extended Thomas Fermi (ETF) approximation ; the kinetic energy densities $\tau_{q}$
are approximated as follows:

$$
\tau_{\mathrm{q}}=\frac{3}{5}\left(3 \pi^{2}\right)^{3 / 2} \rho^{5 / 3}+\frac{1}{18} \frac{\left|\nabla \rho_{\mathrm{q}}\right|^{2}}{\rho_{\mathrm{q}}}+\frac{1}{3} \Delta \rho_{\mathrm{q}} \quad \mathrm{q}=\mathrm{n,p}
$$

We then solve numerically the two coupled Euler Lagrange equations (in $\rho_{n}$ and $\rho_{p}$ ), using a pseudo linearization method analog to that used in ref./4/.

\section{III - 2. Results}

The results are illustrated in Fig. 1 . We have calculated $\sigma_{\delta}$ using 3 different methods. The points correspond to the quantity $\frac{\sigma(I)-\sigma(0)}{I^{2}}$, the crosses to $-\frac{\Sigma(I)-\Sigma(0)}{I^{2}}$ The three extrapolated values for $\mathrm{I}^{2} \rightarrow 0$ agree with in $1 \%$. 0bvious $7 y$ the calculation using the neutron skin is much more accurate. It only requires a first order derivative.

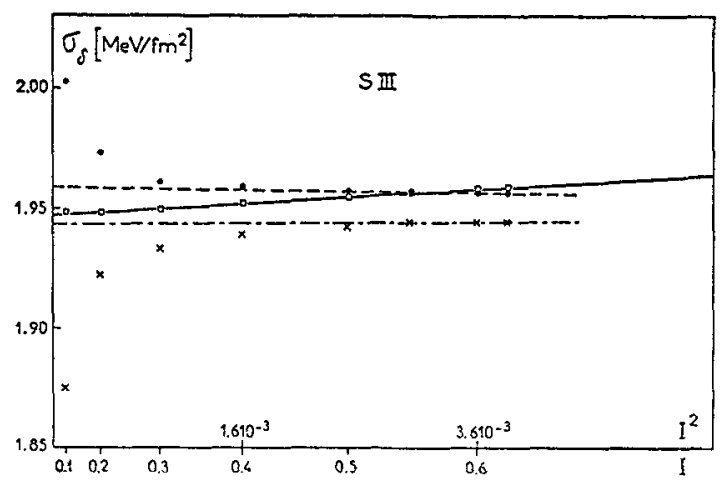

Fig. 1 - Calculation of the surface symmetry tension

Performing such a calculation for various Skyrme forces, we obtained the results of Table 1.

\begin{tabular}{|l|c|c|c|c|c|c|c|c|}
\hline & SII & SIII & SIV & SV & SVI & Ska & SkM & SkM \\
\hline$-\varepsilon_{\text {SS }}[$ MeV] & 59.4 & 35.5 & 67.3 & 95.6 & 27.9 & 75.8 & 56.4 & 55.3 \\
\hline
\end{tabular}

Table 1 - Surface symmetry coefficient for various Skyrme forces (Spin-Orbit force is included). 


\section{IV - THE CASE FOR FINITE NUCLEI}

From the theoretical point of view two problems arise i) as already explained the existence of the neutron skin will induce a decrease of the local asymmetry, $\bar{\delta}$, at the center $(\bar{\delta}<\mathrm{I})$; $i i)$ since the Coulomb force does not saturate, it can only be treated perturbatively. Indeed the densities though smooth or smoothed are not decreasing functions of $r$, and the sharp equivalent radi $i$ cannot any more be calculated from $\rho_{\mathrm{q}}(0)$.

In the absence of the coutomb force, using a demonstration similar to the previous one, we have shown that

$$
\frac{\bar{\delta}}{I}=\frac{1}{1+4 \pi \frac{R^{2}}{A}} \frac{\sigma_{\delta}}{J-L \varepsilon}
$$

where $R$ is the equivalent sharp radius, $A$ the mass number and $L \varepsilon$ a small correction to $J$ because the density at the center is no more $\rho_{n m}$ but $\rho_{0}=\rho_{n m}(1-3 \varepsilon)$. $\left(L=3 \rho_{n m} \frac{\partial \varepsilon_{\delta}(\rho)}{\partial \rho}\right)$. Eq.(20) is the D.M. formula in the finite case, but its derivation was not so straightforward as in the semi infinite case. Somehow one has now i) to introduce the fact that $b / R$ is a small quantity as compared to 1 ( $b$ is the surface thickness), $i i)$ to neglect $\rho^{\prime \prime}(0)$ compared to $\rho(0) / R^{2}$, $i i i$ ) to write the symmetry energy of the finite nucleus

$$
\frac{1}{2} \frac{d^{2}}{d I^{2}} \frac{E}{A}=\frac{\overline{\delta^{2}}}{I^{2}}\left(J-L \varepsilon+4 \pi \frac{R^{2}}{A} \sigma_{\delta}\right)
$$

The knowledge of $\frac{\bar{\delta}}{I}$ gives directly $t$ because of the conservation of $N-Z$

$$
t=\frac{2}{3} I\left(1-\frac{\bar{\delta}}{I}\right) r_{0} A^{1 / 3}
$$

Table 2 displays our preliminary results with a self consistent calculation done as in the previous case but for a finite mass number

\begin{tabular}{|c|c|c|c|}
\hline$A$ & $\infty$ & 450 & 208 \\
\hline$\left(\frac{\bar{\delta}}{\mathrm{I}}\right)_{\mathrm{DM}}$ & 1 & .86 & .83 \\
\hline$\left(\frac{\delta}{\mathrm{I}}\right)_{\mathrm{SC}}$ & 1 & .85 & .80 \\
\hline$\left(\frac{\mathrm{t}}{\mathrm{I}}\right)_{\mathrm{DM}}[\mathrm{fm}]$ & .95 & .82 & .79 \\
\hline$\left(\frac{\mathrm{t}}{\mathrm{I}}\right)_{\mathrm{SC}}[\mathrm{fm}]$ & .95 & .88 & .93 \\
\hline
\end{tabular}

Table 2 - Comparison between (D.M.) predictions and self-consistent calculation.

The subscript (DM) indicates the predictions of the D.M., (sc) the result of the self consistent calculation.

We observe a growing disagreement between DM and SC calculations as $A^{-\frac{1}{3}}$ increases. Obviously a small error on $\frac{\delta}{\mathrm{I}}$ induces a larger one on $\frac{\mathrm{t}}{\bar{I}}$ (see Eq.(22)) 


$$
\frac{\Delta \frac{\mathrm{t}}{\mathrm{I}}}{\frac{\mathrm{t}}{\mathrm{I}}} \simeq \frac{\Delta \frac{\delta}{\mathrm{I}}}{1-\frac{\bar{\delta}}{\mathrm{I}}}
$$

because $1-\frac{\bar{\delta}}{\mathrm{I}}$ is smal1. Indeed, as $A^{1 / 3}$ becomes larger, the relation $(20)$ is better satisfied, but the numerical verification is more delicate. We leave for a further work the point whether or not this disagreement is indicative of a default of the D.M. theory.

\section{$V$ - CONCLUDING REMARKS}

1. In the semi infinite medium, the validity of the D.M. concerning the neutron skin is proved in the framework of very general conditions.

2. A clear distinction must be made between the surface tension of the system as a whole (i.e., with the constraint insuring $i$ ts asymmetry) and the tension of the surface itself.

3. The proof of the corresponding formula in the finite case, even without coulomb force, seems to require some additional hypothesis.

4. The derivation of the sharp radii is not strajghtforward at all : one has to smooth it and to treat properly the coulomb effect. This cannot be done simply by using the "best Fermi" function $/ 5 /$.

Indeed, the D.M. Mode 1 is a theory and as such cannot be seriously disregarded without theoretical criticism. Whether this is, or not, the case remains. so far unclear for us. But it is the only way to learn something.

\section{REFERENCES}

/1/ MYERS W. and SWIATECKI W., Ann. Phys. 55 (1969) 395.

12/ TONDEUR F., J. Phys. G6 (1980) L.71. PEARSON J.M., Nuc7. Phys. A376 (1982) 601.

/3/ KRIVINE H. and TREINER J., Phys. Lett. 124B (1983) 127.

/4/ BOHIGAS 0., CAMPI X., KRIVINE H., TREINER J., Phys. Lett. 64 (1976) 381.

/5/ TREINER J. and KRIVINE H., IPNO/TH 84-1. 